\title{
Brown-York charges at null boundaries
}

\author{
Venkatesa Chandrasekaran, ${ }^{a, b}$ Éanna É. Flanagan, ${ }^{c}$ Ibrahim Shehzad $^{c}$ \\ and Antony J. Speranza ${ }^{d, e}$ \\ ${ }^{a}$ Berkeley Center for Theoretical Physics, University of California, \\ Berkeley, CA 94720, U.S.A. \\ ${ }^{b}$ Institute for Advanced Study, \\ Princeton, NJ 08540, U.S.A. \\ ${ }^{c}$ Department of Physics, Cornell University, \\ Ithaca, NY 14853, U.S.A. \\ ${ }^{d}$ Perimeter Institute for Theoretical Physics, \\ 31 Caroline St. N, Waterloo, ON N2L 2Y5, Canada \\ ${ }^{e}$ Department of Physics, University of Illinois, \\ Urbana-Champaign, Urbana, IL 61801, U.S.A. \\ E-mail: venchandrasekaran@ias.edu, eef3@cornell.edu, \\ is354@cornell . edu, asperanz@gmail.com
}

ABSTRACT: The Brown-York stress tensor provides a means for defining quasilocal gravitational charges in subregions bounded by a timelike hypersurface. We consider the generalization of this stress tensor to null hypersurfaces. Such a stress tensor can be derived from the on-shell subregion action of general relativity associated with a Dirichlet variational principle, which fixes an induced Carroll structure on the null boundary. The formula for the mixed-index tensor $T^{i}{ }_{j}$ takes a remarkably simple form that is manifestly independent of the choice of auxiliary null vector at the null surface, and we compare this expression to previous proposals for null Brown-York stress tensors. The stress tensor we obtain satisfies a covariant conservation equation with respect to any connection induced from a rigging vector at the hypersurface, as a result of the null constraint equations. For transformations that act covariantly on the boundary structures, the Brown-York charges coincide with canonical charges constructed from a version of the Wald-Zoupas procedure. For anomalous transformations, the charges differ by an intrinsic functional of the boundary geometry, which we explicity verify for a set of symmetries associated with finite null hypersurfaces. Applications of the null Brown-York stress tensor to symmetries of asymptotically flat spacetimes and celestial holography are discussed.

KEYwords: Classical Theories of Gravity, Gauge-gravity correspondence

ArXiv EPrint: 2109.11567 


\section{Contents}

1 Introduction $\quad 1$

1.1 Notation 4

2 Timelike boundary $\quad 4$

3 Null boundary $\quad 7$

3.1 Geometry of null surfaces 8

$\begin{array}{lll}3.2 & \text { Null Brown-York stress tensor } & 11\end{array}$

3.3 Comparison to other null Brown-York stress tensors 14

4 Anomalous transformations 18

5 Discussion and future work $\quad 20$

A Connections for Carroll geometries $\quad 22$

\section{Introduction}

Diffeomorphism invariance is a defining feature of gravitational theories such as general relativity, giving rise to charges that comprise an important set of observables in these theories. Although diffeomorphisms supported only in the bulk are pure gauge and hence associated with vanishing charges, transformations that act on the boundary of a spacetime manifold or subregion yield nontrivial charges that provide notions of energy and angular momentum in the region, including contributions from the gravitational field. These charges have found applications in a number of recent works, including symmetries of asymptotically flat space [1-10], asymptotic observables in holography and AdS/CFT [1113], soft hair for black hole horizons and its relation to the information problem [14-21], and edge modes and entanglement for subregions [22-28].

When dealing with subregions bounded by a finite, timelike hypersurface, the construction of Brown and York [29] gives a prescription for determining the gravitational charges in terms of the variational principle for the subregion. By taking the on-shell subregion action $S^{\mathrm{cl}}\left[h_{i j}\right]$ to be a functional of the induced metric $h_{i j}$ on the boundary, one can define a boundary stress tensor in the usual way as the functional derivative $T^{i j}=\frac{2}{\sqrt{-h}} \frac{\delta S^{\mathrm{cl}}}{\delta h_{i j}}$. Given an infinitesimal boundary diffeomorphism generated by a vector $\xi^{i}$, an associated boundary current can be formed using the stress tensor according to the formula $j_{\xi}=T^{i}{ }_{j} \xi^{j} \eta_{i}$, where $\eta_{i}$ is the volume form on the boundary. Integrating this current over a cut of the boundary yields the gravitational charge, and by choosing the vector field $\xi^{i}$ appropriately, one obtains in this way the Brown-York quasilocal energy and angular momentum. 
These charges can be shown to agree with canonical charges generating the associated symmetry transformations on the gravitational phase space [11-13]. This agreement holds when imposing Dirichlet boundary conditions to define the subregion phase space, or more generally for charges constructed using the Wald-Zoupas procedure [30] with a Dirichlet form of the flux [18]. Since the Wald-Zoupas construction deals with open Hamiltonian systems associated with subregions, the charges obtained are colloquially referred to as "nonintegrable," which, more precisely, means that the transformations they generate on phase space do not reproduce the action of their associated diffeomorphism. Generally, the Wald-Zoupas procedure suffers from a number of ambiguities related to the nonintegrability of Hamilton's equation for the diffeomorphism transformation, but these ambiguities can be resolved by demanding a Dirichlet form for the nonintegrable contribution [18, 31]. Although the Brown-York charges appear to sidestep these subtleties involving integrability, the fact that they agree with Wald-Zoupas charges with the Dirichlet flux condition demonstrates that they are simply employing the same resolution to the integrability problem. ${ }^{1}$ In addition to providing a means for constructing canonical charges, the boundary stress tensor $T^{i j}$ also features prominently in holographic dualities such as AdS/CFT, where it is interpreted as the stress tensor operator of the dual conformal field theory [32-34].

In many cases of interest, including exteriors of black hole event horizons, entanglement wedges in holographic setups, and asymptotically flat spacetimes, one is interested in subregions bounded by null hypersurfaces, as opposed to timelike ones. A natural question arises as to whether the Brown-York procedure can be generalized to accommodate null hypersurfaces in order to obtain gravitational charges in this context. The goal of the present paper is to answer this question in the affirmative, and to derive an explicit expression for the null analog of the Brown-York stress tensor for general relativity. The stress tensor has the surprisingly simply expression

$$
T_{j}^{i}=-\frac{1}{8 \pi G}\left(W_{j}^{i}-W \delta^{i}{ }_{j}\right),
$$

where the shape operator $W^{i}{ }_{j}$, defined in (3.4), is the null surface analog of the mixed-index extrinsic curvature $K^{i}{ }_{j}$ of a timelike hypersurface. In fact, the null stress tensor (1.1) depends on $W_{j}^{i}$ in precisely the same way as the standard Brown-York stress tensor depends on $K^{i}{ }_{j}$, making the analogy quite sharp.

The expression (1.1) is obtained by considering the variational principle for general relativity in a subregion bounded by a null hypersurface. This variational principle requires a notion of Dirichlet boundary conditions for the null surface in order to write the subregion action as a functional of boundary geometric data. Unlike the timelike case, where the intrinsic geometry is naturally that of a pseudo-Riemannian structure associated with the induced metric $h_{i j}$, there are a number of different choices for how to define the intrinsic quantities of the null surface that are fixed in a Dirichlet variational principle. The choice

\footnotetext{
${ }^{1}$ In the Brown-York context, instead of finding that Hamilton's equations are not integrable, one instead sees that the subregion action is not stationary for perturbations involving nonzero $\delta h_{i j}$. Demanding that the subregion action be stationary except for terms involving $\delta h_{i j}$ is equivalent to the Dirichlet flux condition for the Wald-Zoupas charges [31].
} 
leading to (1.1) comes from imbuing the null boundary with a Carrollian structure, consisting of a degenerate metric $q_{i j}$ and a preferred null generator $n^{i}$ satisfying $n^{i} q_{i j}=0$ [35-39]. This structure arises naturally from the spacetime geometry after fixing a preferred normal $n_{a}$ to the null surface. The variational principle for general relativity with this boundary condition was explored extensively in [18], and utilizes a null analog of the GibbonsHawking-York boundary term that has appeared in a number of recent works [40-44].

Previously there have been two other proposals for a null Brown-York stress tensor, put forward by Jafari [45] and Donnay and Marteau [46], each of which differs from the expression (1.1). The discrepancies are due to the different choices of geometric structures to associate with the null surface and the corresponding differences in boundary conditions to employ when defining the subregion variational principle. Jafari's construction utilizes a spacelike foliation of the null surface in lieu of a preferred normalization of the null generator. Donnay and Marteau obtain their stress tensor using a null-limit of timelike hypersurfaces, which induces a scalar function on the null surface that can be interpreted as a local surface gravity. In section 3.3, we describe the precise relation between the different null Brown-York stress tensors, and examine how they arise from these different choices of geometric structures on the null surface.

The demonstration of the equivalence between Brown-York and canonical charges requires the stress tensor to satisfy a conservation equation. In the timelike case, this conservation equation simply states that the stress tensor is divergenceless with respect to the unique connection $D_{i}$ compatible with the induced metric. Null surfaces are more subtle in this regard, since a Carrollian structure does not determine a unique connection with respect to which to define the covariant conservation of $T_{j}^{i}$. Nevertheless, we show in section 3.1 that there is a class of torsion-free, but generically not metric compatible, connections associated with the Carroll structure that are naturally induced from the spacetime Levi-Civita connection as rigged connections, using a construction of Mars and Senovilla [47]. While such connections have appeared previously in describing null infinity and finite null surfaces embedded in spacetime [48-50], they have not been considered in the recent literature on Carroll geometry (see, however, [51]), and we comment on their main properties in appendix A. We further show that the conservation of the stress tensor with respect to any such connection is equivalent to the constraint equations of general relativity on the null surface, consisting of the Raychaudhuri and Damour-Navier-Stokes equations $[52,53]$. The connection between the gravitational constraint equations and conservation laws was also explored by Donnay and Marteau for their null-limit stress tensor [46], and in section 3.3 we compare their conservation equation to the one obtained for the stress tensor in the present work.

After demonstrating the equivalence between the null Brown-York charges and canonical charges for transformations that act covariantly on the Carrollian geometry, we turn our attention in section 4 to so-called anomalous transformations. These arise from bulk diffeomorphisms that do not fully preserve the fixed null normal $n_{a}$, and were shown in [18] to be the essential feature determining extensions of gravitational charge algebras, which, when evaluated on a black hole horizon, lead to information about the horizon entropy. 
We demonstrate that for such anomalous transformations, the Brown-York and canonical charges in general do no agree, instead differing by a functional of the intrinsic geometry. We explicitly exhibit this difference by comparing the Brown-York expression to the canonical charges associated with BMS-like transformations on finite null surfaces that were obtained by Chandrasekaran, Flanagan, and Prabhu [50].

We conclude in section 5 with some discussions on potential applications to symmetries of asymptotically flat space, celestial holography, and the fluid-gravity correspondence, and comment on some directions for future work.

\subsection{Notation}

Latin letters from the beginning of the alphabet $a, b, c, \ldots$ are used to denote spacetime tensor indices, while those from the middle of the alphabet $i, j, k, \ldots$ are used for tensors defined on a timelike or null bounding hypersurface. Differential forms such as the spacetime volume form $\epsilon$ or hypersurface volume form $\eta$ are often written with indices suppressed. When denoting a contraction on one or more indices, we will use the shorthand $\epsilon_{a}$ to indicate the indices which are contracted, while continuing to suppress the remaining indices. We also use the notation $i_{V}$ for contraction with a vector $V^{a}$ into a differential form.

\section{Timelike boundary}

We begin by reviewing the construction of Brown-York charges for timelike boundaries, and the argument demonstrating their equivalence to canonical charges. This argument is familiar from previous considerations regarding so-called "counterterm subtraction charges" in asymptotically anti-de Sitter spaces $[11,12]$ and also discussions of integrable charges for finite timelike boundaries [13].

Given an open subregion $\mathcal{U}$ bounded in spatial extent by a timelike surface $\mathcal{T}$, the action for the subregion is given by a sum of a bulk Einstein-Hilbert term and the boundary Gibbons-Hawking-York term, ${ }^{2}$

$$
\begin{aligned}
S & =\int_{\mathcal{U}} L-\int_{\mathcal{T}} \ell \\
L & =\frac{1}{16 \pi G}(R-2 \Lambda) \epsilon \\
\ell & =-\frac{1}{8 \pi G} K \eta,
\end{aligned}
$$

where $\epsilon$ is the spacetime volume form, $R$ is the spacetime Ricci scalar, and $K$ is the trace of the extrinsic curvature of $\mathcal{T}$. Also $\eta$ is the induced volume form on $\mathcal{T}$, defined such that $\epsilon \stackrel{\mathcal{T}}{=}-n \wedge \eta$, where $n_{a}$ is the outward pointing unit normal to $\mathcal{T}$. The orientation of $\mathcal{T}$ is

\footnotetext{
${ }^{2}$ We leave out contributions from future or past boundaries and codimension-2 corners, which are not needed in obtaining the Brown-York charges.
} 
chosen to be that determined by $\eta .^{3}$ The boundary term $\ell$ is chosen so that the action satisfies a Dirichlet variational principle with respect to the induced metric $h_{i j}$ on $\mathcal{T}$. Its variation is given by (see e.g. $[13,54]$ )

$$
\delta S=\int_{\mathcal{U}} E^{a b} \delta g_{a b}+\int_{\mathcal{T}}\left(\pi^{i j} \delta h_{i j}-d \beta\right),
$$

where $E^{a b}=0$ are the vacuum Einstein field equations, the momenta $\pi^{i j}$ are given by

$$
\pi^{i j}=-\frac{1}{16 \pi G}\left(K^{i j}-K h^{i j}\right) \eta
$$

and $\beta$ contributes terms that localize to the past and future boundaries of $\mathcal{T}$; explicitly, it is given by

$$
\beta=\frac{1}{16 \pi G}\left(g^{a b}-n^{a} n^{b}\right) n^{c} \delta g_{b c} \eta_{a},
$$

although we will drop these terms since we are ignoring contributions from future and past boundaries.

The action $S$ is therefore stationary ${ }^{4}$ when the bulk equations of motion hold and the induced metric $h_{i j}$ is fixed. This then allows us to view the classical action as a functional of $h_{i j}, S^{\mathrm{cl}}\left[h_{i j}\right]$, and the boundary stress tensor is given by the variation of this functional with respect to $h_{i j}$,

$$
T^{i j}=\frac{2}{\sqrt{-h}} \frac{\delta S^{\mathrm{cl}}}{\delta h_{i j}}=-\frac{1}{8 \pi G}\left(K^{i j}-K h^{i j}\right) .
$$

This stress tensor can be used to construct boundary Noether currents associated with infinitesimal diffeomorphisms that preserve the hypersurface $\mathcal{T}$. These are generated by vector fields $\xi^{a}$ tangent to $\mathcal{T}$, and if $\xi^{i}$ is the restriction of the vector to $\mathcal{T}$, the current is given by $^{5}$

$$
j_{\xi}=-T_{j}^{i} \xi^{j} \eta_{i}
$$

The Noether current is conserved when $\xi^{i}$ generates a symmetry of the boundary metric, which can be seen by computing its divergence,

$$
d j_{\xi}=-\eta\left(T^{i j} D_{i} \xi_{j}+D_{i} T_{j}^{i} \xi^{j}\right)=-\frac{\eta}{2} T^{i j} £_{\xi} h_{i j}-(\operatorname{div} T)_{j} \xi^{j} \eta
$$

\footnotetext{
${ }^{3}$ This sign convention for $\eta$ is opposite to that used in reference [13], and is opposite the orientation induced on $\mathcal{T}$ when viewed as a component of $\partial \mathcal{U}$. This implies that Stokes theorem for the subregion takes the form $\int_{\mathcal{U}} d \alpha=-\int_{\mathcal{T}} \alpha$ (dropping contributions from other components of $\partial \mathcal{U}$ ). This orientation for $\mathcal{T}$ ensures that Stokes theorem for integrals over a segment of $\mathcal{T}_{1}^{2}$ between two cuts $\mathcal{S}_{1}$ and $\mathcal{S}_{2}$ of $\mathcal{T}$, with $\mathcal{S}_{1}$ to the past of $\mathcal{S}_{2}$ takes the form $\int_{\mathcal{T}_{1}^{2}} d \alpha=\int_{\mathcal{S}_{2}} \alpha-\int_{\mathcal{S}_{1}} \alpha$, where the orientations of $\mathcal{S}_{1,2}$ are those induced by viewing them as the boundary of bulk spacelike hypersurfaces $\Sigma_{1,2}$. Hence, if we take $\mathcal{U}_{1}^{2}$ to be the region of $\mathcal{U}$ bounded spatially by $\mathcal{T}_{1}^{2}$ and to the past and future by $\Sigma_{1,2}$, these conventions imply that $\partial \mathcal{U}_{1}^{2}=-\mathcal{T}_{1}^{2}+\Sigma_{2}-\Sigma_{1}$ (where the sign indicates the relative orientations), $\partial \Sigma_{1,2}=\mathcal{S}_{1,2}$, and $\partial \mathcal{T}_{1}^{2}=\mathcal{S}_{2}-\mathcal{S}_{1}$.

${ }^{4} \mathrm{Up}$ to contributions from future and past boundaries.

${ }^{5}$ The sign in this equation is somewhat nonstandard, and arises due to the choice of orientation of $\mathcal{T}$. The stress tensor (2.7) is insensitive to the choice of orientation, since it arises from the $\int_{\mathcal{T}} \pi^{i j} \delta h_{i j}$ term in (2.4) after stripping off the volume form $\eta$ and the integral over $\mathcal{T}$. Since under a change in orientation $\eta \rightarrow-\eta$ and $\int_{\mathcal{T}} \rightarrow-\int_{\mathcal{T}}$, these signs cancel out in the definition of $T^{i j}$. However, the current $j_{\xi}$ is constructed by contracting with the volume form $\eta$, and hence it flips sign under a change in orientation. As we will see below, the current that reproduces the canonical charges is the one associated with the orientation on $\mathcal{T}$ naturally induced as a component of $\partial \mathcal{U}$. The volume form for this induced orientation is $-\eta$, which accounts for the sign in (2.8).
} 
where $D_{i}$ is the connection compatible with $h_{i j}$. The second term in this expression involving $\operatorname{div} T$ is recognized as the momentum constraint of general relativity associated with the hypersurface $\mathcal{T}$, and hence vanishes on-shell. This is a feature that will continue to hold in the null case: the covariant conservation of the Brown-York stress tensor is equivalent to imposing the momentum constraints of vacuum general relativity on $\mathcal{T}$. When matter is present, there would be an additional boundary contribution from the matter stress tensor so that the total boundary stress tensor is conserved. The first term in (2.9) clearly vanishes when $\xi^{i}$ is a Killing vector for $h_{i j}$, and hence in this case $j_{\xi}$ defines a conserved current.

Even when $\xi^{i}$ does not generate a symmetry of the boundary metric, the current $j_{\xi}$ defines an important quantity due to its relation to gravitational charges constructed using canonical methods. In particular, the Wald-Zoupas procedure [30, 50, 55] employs covariant phase space techniques to construct charges that generically are not conserved due to the presence of fluxes through the subregion boundary $\mathcal{T}$. Instead of a conservation equation, these charges satisfy a continuity equation where the change in the charge is related to a well-defined flux. Utilizing the reformulation of the Wald-Zoupas procedure given in references [18, 31], which applies the techniques developed by Harlow and Wu [13] for dealing with boundaries in the covariant phase space, the gravitational charges can be defined as the integral of a current $h_{\xi}$ over a cut of the boundary $\mathcal{T}$. The explicit expression for $h_{\xi}$ is

$$
h_{\xi}=Q_{\xi}+i_{\xi} \ell-\beta\left[£_{\xi} g_{a b}\right],
$$

where

$$
Q_{\xi}=-\frac{1}{16 \pi G} \epsilon_{a b} \nabla^{a} \xi^{b}
$$

is the Noether potential, $\ell$ is the boundary term (2.3), and $\beta\left[£_{\xi} g_{a b}\right]$ is the quantity (2.6) evaluated with $\delta g_{a b}=£_{\xi} g_{a b}$. Using that the boundary term $\ell$ transforms covariantly under any diffeomorphism generated by a vector field $\xi^{a}$ that is tangent to $\mathcal{T}$, one can derive a continuity equation for $h_{\xi}$ of the form

$$
d h_{\xi}=-\pi^{i j} £_{\xi} h_{i j},
$$

where the expression on the right represents the flux density through the boundary $\mathcal{T}$.

Since $\pi^{i j}=\frac{1}{2} \eta T^{i j}$, comparing to equation (2.9) and imposing the constraint $\operatorname{div} T=0$ allows us to conclude

$$
d h_{\xi}=d j_{\xi}
$$

Hence the charge densities $h_{\xi}$ and $j_{\xi}$ can differ at most by a closed form $s_{\xi}$. Furthermore, since $h_{\xi}$ and $j_{\xi}$ are covariantly constructed from $\xi^{i}$ and the boundary fields for arbitrary choices of $\xi^{i}$, we can apply a theorem due to Wald [56] to conclude that $s_{\xi}$ is exact, $s_{\xi}=d c_{\xi}$. This then implies that the charges obtained by integrating the charge densities over a cut $\mathcal{S}$ of $\mathcal{T}$ are insensitive to the choice of $c_{\xi}$, implying that the Wald-Zoupas and Brown-York charges coincide,

$$
H_{\xi}=\int_{\mathcal{S}} h_{\xi}=\int_{\mathcal{S}} j_{\xi} \equiv \mathcal{Q}_{\xi}^{\mathrm{BY}} .
$$

Finally, as discussed in refs. $[12,31,32,34,57,58]$, when taking a limit of the surface $\mathcal{T}$ to an asymptotic boundary, additional boundary terms $\ell_{\text {ct }}$ must be added to the action 
in order to obtain finite charges in a process known as holographic renormalization. These additional counterterms are required to be covariant functionals of the intrinsic geometry in order to preserve the Dirichlet form of the variational principle. Such terms then change $\pi^{i j}$ by the variational derivative $\pi_{\mathrm{ct}}^{i j}=\frac{\delta \ell_{\mathrm{ct}}}{\delta h_{i j}}$. Covariance of $\ell_{\mathrm{ct}}$ is crucial for ensuring that the new stress tensor continues to be conserved, which requires $D_{i} \pi_{\mathrm{ct}}^{i j}=0$. This conservation equation is simply the Noether identity associated with the covariant functional $\ell_{\mathrm{ct}}$, which holds identically due to invariance of $\ell_{\text {ct }}$ under boundary diffeomorphisms [59-61]. Hence, we can conclude that the equality of Wald-Zoupas and Brown-York charges is unaffected by the process of holographic renormalization, provided the boundary counterterms are covariant functionals of the intrinsic boundary geometry. ${ }^{6}$ Note that subtleties can arise for transformations that are only tangential to $\mathcal{T}$ asymptotically, as occurs for some asymptotic symmetry transformations. These can induce anomalous transformations on $\ell_{\text {ct }}$ and the intrinsic quantities on $\mathcal{T}$, due to the nonzero transverse component of the vector field. In these cases, the canonical and Brown-York charges can differ by terms related to holographic Weyl anomalies [12, 32, 34, 57, 62, 63]. A related example of this effect that occurs for null surfaces is examined in section 4 .

\section{Null boundary}

We can now repeat this analysis for a subregion bounded by a null hypersurface $\mathcal{N}$. The main subtlety is that the intrinsic geometry is no longer characterized by a pseudoRiemannian structure, involving a nondegenerate metric. Instead, the geometry consists of a degenerate metric $q_{i j}$ with a single null direction, and a preferred generator $n^{i}$ of the kernel of $q_{i j}$, i.e. a vector satisfying $n^{i} q_{i j}=0$. Together, these objects define a (weak) Carroll structure for the null surface $\mathcal{N}$ [35-39]. While the degenerate metric $q_{i j}$ is naturally induced as the pullback of the spacetime metric to $\mathcal{N}$, the additional structure encoded in $n^{i}$ arises after choosing a distinguished normal form $n_{a}$ of the null surface, after which $n^{i}$ is determined by raising the index with $g^{a b}$ and restricting the vector to $\mathcal{N}$. These structures were argued in [18] to be the natural quantities with respect to which to formulate the Dirichlet variational principle for null boundaries.

There are two main subtleties associated with working with a Carrollian, as opposed to a pseudo-Riemannian, structure. First, the process of lowering indices with the degenerate intrinsic metric does not produce an isomorphism between tangent and cotangent vectors, and hence the index placement for tensors becomes important. Second, there is no preferred connection available for defining covariant derivatives of tensors. Despite these complications, we will find that a null version of the Brown-York stress tensor $T^{i}{ }_{j}$ can be obtained independent of any choice of connection. It is naturally defined with one contravariant and one covariant index, as is appopriate when viewing the stress tensor as a linear map from vectors $\xi^{i}$ into their associated charge densities $j_{\xi}=-T_{j}^{i} \xi^{j} \eta_{i}$, with $\eta_{i}$ the volume form [64]. Furthermore, a connection-independent notion of the covariant conservation of

\footnotetext{
${ }^{6}$ These anomalous transformations manifest as a mismatch between the transformation of $\ell_{\text {ct }}$ on the phase space and its transformation under the Lie derivative, $\delta_{\xi} \ell_{c t} \neq £_{\xi} \ell_{c t}$.
} 
the stress tensor $(\operatorname{div} T)_{j}=0$ also arises from the definition, and this condition turns out to precisely coincide with the imposition of the constraint equations on the null surface.

\subsection{Geometry of null surfaces}

In order to describe the Dirichlet variational principle with a null boundary, we need to review a few details on the intrinsic and extrinsic geometry of null surfaces. The degenerate metric $q_{i j}$ determines a spatial volume form $\mu$ (up to a sign, which can be fixed by a choice of orientation), which is a horizontal form of maximal degree, meaning it is one degree below a top form and $i_{n} \mu=0$. This spatial volume form is such that on any codimension- 1 cut of $\mathcal{N}, \mu$ pulls back to the induced volume form compatible with the pullback of $q_{i j}$ and the chosen orientation of the cut. The preferred null generator $n^{i}$ also determines a volume form $\eta$ on the full null surface, which is the unique top form that satisfies $i_{n} \eta=\mu$. These structures then determine a set of first order differential invariants, the expansion $\Theta$, extrinsic curvature $K_{i j},{ }^{7}$ and shear $\sigma_{i j}$ according to the equations

$$
\begin{aligned}
d \mu & =\Theta \eta \\
K_{i j} & =\frac{1}{2} £_{n} q_{i j} \\
\sigma_{i j} & =K_{i j}-\frac{1}{n-1} \Theta q_{i j} .
\end{aligned}
$$

where $n=d-1$ is the dimension of the null hypersurface.

The extrinsic geometry is characterized by the shape operator or Weingarten map $W_{j}^{i}$ of the null surface, which is determined after fixing a preferred spacetime 1-form $n_{a}$ at $\mathcal{N}$ to serve as the null normal. Letting $\Pi^{a}{ }_{i}$ denote the pullback map to $\mathcal{N}$, we note that the spacetime covariant derivative of the null normal $\nabla_{a} n^{b}$ upon taking a pullback produces a tensor $\Pi^{a}{ }_{i} \nabla_{a} n^{b}$ whose $b$ index is tangential. This therefore defines a tensor $W_{j}^{i}$ on $\mathcal{N}$ which we refer to as the shape operator; explicitly it can be defined as the unique tensor satisfying

$$
W_{j}^{i} \Pi_{i}^{b}=\Pi_{j}^{a} \nabla_{a} n^{b} .
$$

Some components of the shape operator are determined by the intrinsic geometry of $\mathcal{N}$; in particular, the extrinsic curvature is obtained by lowering an index with $q_{i j}$,

$$
W_{j}^{i} q_{i k}=K_{k j} .
$$

The remaining components involve terms in $W_{j}^{i}$ of the form $n^{i} \rho_{j}$, which do not contribute to equation (3.5). One such component arises from the equation

$$
W_{j}^{i} n^{j}=k n^{i},
$$

which holds since $n^{a}$ is parallel to null geodesics in spacetime. Hence, $n^{i}$ is an eigenvector of the shape operator, and its eigenvalue $k$ is called the inaffinity.

\footnotetext{
${ }^{7}$ Despite this terminology, the "extrinsic curvature" $K_{i j}$ is fully determined by the intrinsic quantities $\left(q_{i j}, n^{i}\right)$.
} 
To be more explicit about the decomposition of the shape operator, we need to introduce an auxiliary one-form $l_{i}$, normalized relative to the null generator by $n^{i} l_{i}=-1$. Note that there is no preferred choice for $l_{i}$ for a generic null surface. Intrinsically, $l_{i}$ defines an Ehreshmann connection [65, 66], which is just a projector $s^{i}{ }_{j}=-n^{i} l_{j}$ onto vertical vectors parallel to $n^{i}$. This similarly allows us to define a projector $q_{j}^{i}$ onto horizontal forms through the relation

$$
q_{j}^{i}=\delta_{j}^{i}+n^{i} l_{j}
$$

as well as a partial inverse $q^{i j}$ of the degenerate metric $q_{j k}$ through the relations $q^{i j} q_{j k}=q_{k}^{i}$, $q^{i j} l_{j}=0$. From the extrinsic perspective, $l_{i}$ can be taken to arise from a null rigging vector, which is a transverse vector $l^{a}$ defined on the null surface satisfying $l \cdot n=-1, l \cdot l=0$. This implies that $l^{a}$ is an outward pointing vector at the null surface. Then $l_{i}=\Pi^{a}{ }_{i} l_{a}$ defines the desired one-form on the null surface. Having introduced $l_{i}$, the shape operator can then be decomposed as

$$
\begin{aligned}
W_{j}^{i} & =K^{i}{ }_{j}+n^{i} \rho_{j} \\
\rho_{j} & =\varpi_{j}-k l_{j}
\end{aligned}
$$

where $K^{i}{ }_{j}=q^{i k} K_{k j}, \rho_{j}$ is the rotation one-form defined by,

$$
\rho_{j}=-\Pi^{a}{ }_{j} l_{b} \nabla_{a} n^{b}
$$

and the Hájiček one-form $\varpi_{i}$ is the spatial projection of $\rho_{j}$,

$$
\varpi_{i}=q_{i}^{j} \rho_{j} .
$$

We can also explicitly express the volume form on $\mathcal{N}$ in terms of $l^{a}$ and the spacetime volume form by writing $\eta=\Pi^{*}\left(-i_{l} \epsilon\right)$, where $\Pi^{*}$ indicates a pullback. Note as in the timelike case, the volume form $\eta$ is associated with an orientation of $\mathcal{N}$ that is opposite the natural orientation induced on $\mathcal{N}$ as a component of the boundary of $\mathcal{U}$.

The rigging vector $l^{a}$ also provides a natural projector for spacetime vectors onto the null surface, given by

$$
\Pi_{b}^{a}=\delta_{b}^{a}+l^{a} n_{b},
$$

which then allows us to define an inclusion map $\Pi_{a}^{i}$ that inverts the pullback map for covectors in the sense

$$
\Pi_{b}^{i} \Pi_{i}^{a}=\Pi_{b}^{a} .
$$

We can then use $\Pi_{a}^{i}$ and $\Pi^{b}{ }_{j}$ to map intrinsic tensor fields on the null surface into spacetime tensor fields defined at $\mathcal{N}$.

Additionally, this projector induces a natural rigged connection on the null surface from the spacetime Levi-Civita connection through a construction of Mars and Senovilla [47]. If $V^{a}$ is tangent to $\mathcal{N}$, we can define the rigged covariant derivative as

$$
D_{a} V^{b}=\Pi^{c}{ }_{a} \Pi_{d}^{b} \nabla_{c} V^{d}
$$


which then defines an intrinsic covariant derivative on the vector $V^{i}=\Pi_{a}^{i} V^{a}$ to be

$$
D_{i} V^{j}=\Pi^{a}{ }_{i} \Pi_{b}^{j} D_{a} V^{b}
$$

$D_{i}$ is extended to covectors $U_{i}$ by first mapping it to spacetime using the inclusion map $\Pi_{a}^{i}$, taking the covariant derivative, and then pulling back,

$$
D_{i} U_{j}=\Pi_{i}^{a} \Pi_{j}^{b} \nabla_{a}\left(\Pi_{b}^{k} U_{k}\right)
$$

$D_{i}$ is then extended in the usual way to tensors of arbitrary degree. It is important to emphasize that this intrinsic connection $D_{i}$ depends on the choice of auxiliary one-form $l_{i}$. Note that it is manifestly torsion-free, but generically does not preserve any of the intrinsic structures on the surface. Instead, we have the following relations (derived in appendix A):

$$
\begin{aligned}
D_{i} q_{j k} & =l_{j} K_{i k}+l_{k} K_{i j} \\
D_{i} n^{j} & =W_{i}^{j}=K_{i}^{j}+n^{j} \rho_{i} \\
D_{i} \eta & =-\rho_{i} \eta \\
D_{i} \mu & =K^{j}{ }_{i} \eta_{j}
\end{aligned}
$$

From the intrinsic perspective, these relations can also be used as the definition of a connection compatible with a given Carroll structure and associated Ehresmann connection $l_{i}$. In doing so, the rotation one-form $\rho_{i}$ appears as additional data needed to fully specify the connection, beyond that contained in $\left(q_{i j}, n^{i}, l_{j}\right)$. Additionally, there is a final relation involving $D_{i} l_{j}$ that is not fixed by equations (3.17)-(3.20), as shown in equation (A.8). The data in $D_{i} l_{j}$ that is not fixed by quantities already defined is captured by its symmetric, horizontal component, $\nu_{i j}=q^{m}{ }_{i} q^{n}{ }_{j} D_{(m} l_{n)}$. As equation (3.19) shows, $\rho_{i}$ characterizes the failure of the connection to preserve the volume form. Similarly, $\nu_{i j}$ measures the failure of the connection to preserve the Ehresmann connection $l_{i}$, although there are additional obstructions to the vanishing of $D_{i} l_{j}$ described in appendix A. Intrinsically, one is free to work with a connection that imposes $\rho_{i}=0$ and $\nu_{i j}=0$; however, there are preferred, generically nonzero, choices for these quantities when working with a rigged connection induced from the spacetime connection.

Finally, we mention how to express the divergence of a vector field on the null surface in terms of the connection $D_{i}$. Since the null surface has a preferred volume form $\eta$, the divergence of a vector field $V^{i}$ can be defined independently of a connection through the equation

$$
d i_{V} \eta=(\operatorname{div} V) \eta
$$

Because the connection $D_{i}$ generally does not preserve the volume form, the expression for $\operatorname{div} V$ in terms of $D_{i}$ contains a contribution from $\rho_{i}$,

$$
\operatorname{div} V=D_{i} V^{i}-\rho_{i} V^{i}
$$




\subsection{Null Brown-York stress tensor}

We can now describe the construction of the null boundary Brown-York stress tensor, and demonstrate the equivalence between the charges constructed from it and the canonical charges. Before deriving the result, we first comment on an important point regarding the index placement of the stress tensor we are seeking to obtain. On timelike surfaces, the presence of a nondegenerate metric allows indices to be raised and lowered, and so the tensors $T_{i j}, T^{i j}$ and $T^{i}{ }_{j}$ all contain the same information. This is no longer true on a null surface, and there is a question as to which index placement is correct. The answer is that the stress tensor is naturally defined as a mixed index object, $T^{i}{ }_{j}$. This is because the stress tensor should be viewed as a map from a vector field $\xi^{i}$ to an associated current $j_{\xi}$, which can be integrated over codimension- 1 surfaces inside of $\mathcal{N}$ to obtain fluxes of energy and momentum. This current is obtained by contracting the vector $T^{i}{ }_{j} \xi^{j}$ into the volume form $\eta$ on $\mathcal{N}$ (see related comments in [64]). Note that the presence of a volume form $\eta$ as a natural structure characterizing the geometry of $\mathcal{N}$ is important for obtaining a two-index tensor. This suggests that an even more natural object characterizing the stress-energy of the theory is the covector-valued differential form $T_{j}^{i} \eta_{i}$. However, since there is a preferred volume form when working with a fixed null normal $n_{a}$, we will focus on the associated stress tensor $T_{j}^{i}$.

We begin as before with an open subregion $\mathcal{U}$ in spacetime, now bounded in spatial extent by a null hypersurface $\mathcal{N}$. Nullness of $\mathcal{N}$ is imposed as a boundary condition for the phase space of field configurations, and we further impose that $\mathcal{N}$ be equipped with a preferred null normal that is also fixed when taking variations, $\delta n_{a}=0$. The action is taken to contain the same bulk term (2.2), but in place of the Gibbons-Hawking-York term, the boundary term for the null surface is constructed from the inaffinity $k$ according to ${ }^{8}$

$$
\ell=-\frac{1}{8 \pi G} k \eta,
$$

and the subregion action is defined to be

$$
S=\int_{\mathcal{U}} L-\int_{\mathcal{N}} \ell
$$

where the orientation of $\mathcal{N}$ is again chosen to be opposite the induced orientation as a component of $\partial \mathcal{U}$. The variation of the action with this boundary term takes the form of a Dirichlet variational principle [18],

$$
\delta S=\int_{\mathcal{U}} E^{a b} \delta g_{a b}+\int_{\mathcal{N}}\left(\pi^{i j} \delta q_{i j}+\pi_{i} \delta n^{i}-d \beta\right)
$$

where

$$
\begin{aligned}
\pi^{i j} & =-\frac{1}{16 \pi G}\left(K^{i j}-(\Theta+k) q^{i j}\right) \eta \\
\pi_{i} & =\frac{1}{8 \pi G}\left(\Theta l_{i}+\varpi_{i}\right) \eta \\
\beta & =\frac{1}{16 \pi G}\left(g^{a b} n^{c}-n^{a} g^{b c}\right) \delta g_{a b} \eta_{c} .
\end{aligned}
$$

\footnotetext{
${ }^{8}$ Another choice for the boundary term is $-\frac{1}{8 \pi G}(k+\Theta) \eta[40,43,44]$. The additional term involving $\Theta$ can be shown to be a total derivative on $\mathcal{N}$, and hence only changes the definition of the corner term $\beta$ (see (3.28)), and does not affect the Dirichlet variational principle.
} 
This action is stationary when the bulk equations of motion hold and the intrinsic Carroll structure defined by $\left(q_{i j}, n^{i}\right)$ is held fixed, which allows the classical action to be viewed as a functional of this structure, $S^{\mathrm{cl}}\left[q_{i j}, n^{i}\right]$.

Since the classical action is now a functional of two geometric quantities $\left(q_{i j}, n^{i}\right)$ instead of a single metric $h_{i j}$, defining a stress tensor associated with it requires slightly more care than in the timelike case. In general, the stress tensor should characterize how the action responds to a diffeomorphism acting on the boundary, for which $\delta_{\xi} q_{i j}=£_{\xi} q_{i j}$ and $\delta_{\xi} n^{i}=£_{\xi} n^{i}$. Using the expression (3.25) for a general variation of the action and dropping terms that localize to the boundary of $\mathcal{N}$, we find that $\delta_{\xi} S^{\mathrm{cl}}=-\int_{\mathcal{N}} \mathcal{E}_{\xi}$, with

$$
\mathcal{E}_{\xi}=-\pi^{i j} £_{\xi} q_{i j}-\pi_{i} £_{\xi} n^{i}=-\pi^{i j}\left(\xi^{k} D_{k} q_{i j}+2 D_{i} \xi^{k} q_{k j}\right)-\pi_{i}\left(\xi^{k} D_{k} n^{i}-n^{k} D_{k} \xi^{i}\right),
$$

where $D_{i}$ is taken for the moment to be an arbitrary torsionless affine connection on $\mathcal{N}$. Since the first equality here only involves Lie derivatives, the expression does not depend on the choice of $D_{i}$. The expression on the right hand side of (3.29) can be rearranged to express the equation in the form

$$
\mathcal{E}_{\xi}=d j_{\xi}-f_{\xi},
$$

where $j_{\xi}$ and $f_{\xi}$ are unambiguously determined by requiring that they both depend linearly and algebraically on $\xi^{i}$. We will use this decomposition to define the stress tensor by the relation $j_{\xi}=-T^{i}{ }_{j} \xi^{j} \eta_{i}$ (see footnote 5 regarding this choice of sign), as well as a generalized divergence by the equation $f_{\xi}=-(\operatorname{div} T)_{j} \xi^{j} \eta$. Using the undensitized momenta $\left(p^{i j}, p_{i}\right)$, defined by $\pi^{i j}=\eta p^{i j}, \pi_{i}=\eta p_{i}$, the boundary stress tensor and its generalized divergence are found to be ${ }^{9}$

$$
\begin{aligned}
T_{j}^{i} & =2 p^{i k} q_{k j}-n^{i} p_{j} \\
(\operatorname{div} T)_{j} & =D_{i} T^{i}{ }_{j}-\rho_{i} T^{i}{ }_{j}-p^{i k} D_{j} q_{i k}-p_{i} D_{j} n^{i}
\end{aligned}
$$

Note that the corrections appearing in the generalized divergence are similar to those that occur in the divergence formula (3.22) for a vector field with respect to a connection $D_{i}$ that does not preserve the volume form. Although the expression for $(\operatorname{div} T)_{j}$ appears to depend on the choice of connection, such dependence is superficial, as can be seen by noting that

$$
(\operatorname{div} T)_{j} \xi^{j} \eta=d\left(T^{i}{ }_{j} \xi^{j} \eta_{i}\right)-\pi^{i j} £_{\xi} q_{i j}-\pi_{i} £_{\xi} n^{i}
$$

\footnotetext{
${ }^{9}$ These expressions for a stress tensor and generalized divergence are not special to general relativity, but instead hold for any theory whose action is a functional of a Carroll structure, $S\left[q_{i j}, n^{i}\right]$. The Carrollian momenta $p^{i j}$ and $p_{i}$ can be defined by variational derivatives of such an action with respect to $q_{i j}$ and $n^{i}$, and the stress tensor of the theory is still given by (3.31). This allows generalizations not only to other theories of gravity possessing a Dirichlet variational principle, but also to more general Carrollian field theories defined intrinsically on a null surface (see e.g. [67]). A similar expression for a stress tensor of asymptotically flat 3D gravity in terms of Carrollian momenta was presented in [68]. A related construction of Carrollian momenta and conservation laws was considered in [69], although they utilize a slightly different set of geometric structures and momenta. Their conservation laws are naturally interpreted as a null limit of ordinary covariant conservation with respect to a pseudo-Riemannian connection, as explored in [70, 71].
} 
with all terms on the right hand side manifestly independent of the connection. ${ }^{10}$ The expression (3.31) gives the null version of the Brown-York stress tensor, which can be rearranged using the expressions (3.26) and (3.27) and the decomposition (3.8) of the shape operator to give

$$
T_{j}^{i}=-\frac{1}{8 \pi G}\left(W_{j}^{i}-W \delta^{i}{ }_{j}\right)
$$

where $W=W_{i}^{i}=\Theta+k$. This expression is exactly analogous to the timelike Brown-York stress tensor (2.7), with the null shape operator $W^{i}{ }_{j}$ replacing the extrinsic curvature tensor $K^{i}{ }_{j}$ in the timelike case. In fact, $K^{i}{ }_{j}$ has the interpretation of a shape operator for a timelike surface, which further tightens the analogy between the two cases. An important property of the null Brown-York stress tensor (3.35) is that because it is constructed directly from $W_{j}^{i}$, it is completely independent of the choice of $l_{i}$, despite a superficial dependence on this choice in the expressions for the individual momenta $p^{i j}$ and $p_{i}$. It is worth pointing out that the mixed index structure $T^{i}{ }_{j}$ is important for obtaining an object that is independent of $l_{i}$ : any procedure for raising or lowering an index to obtain tensors $T^{i j}$ or $T_{i j}$ will necessarily introduce dependence on some auxiliary structure such as $l_{i}$, or else kill some components if, for example, the degenerate metric $q_{i j}$ is used to lower an index.

To prove equality between the Brown-York current $j_{\xi}$ and the charge density $h_{\xi}$ constructed using the Wald-Zoupas procedure [18], we must show as in the timelike case that the divergence of the stress tensor (3.32) vanishes as a consequence of the constraint equations on $\mathcal{N}$. To do so, we now take the connection $D_{i}$ to be an induced rigged connection, satisfying equations (3.17)-(3.20). The first term in $\operatorname{div} T$ takes the expected from as a covariant divergence with respect to the connection $D_{i}$. The remaining terms can be shown to cancel:

$$
\begin{aligned}
& 2 \rho_{i} W_{j}^{i}-2 W \rho_{j}+\left(K^{i k}-W q^{i k}\right)\left(l_{i} K_{j k}+l_{k} K_{j i}\right)-2\left(\varpi_{i}+\Theta l_{i}\right) W_{j}^{i} \\
& =-2\left[-\rho_{i} W_{j}^{i}+W \rho_{j}+\rho_{i} W^{i}{ }_{j}+(\Theta+k) l_{i} W_{j}^{i}\right] \\
& =0
\end{aligned}
$$

where we have used that $l_{i} K^{i k}=l_{i} q^{i k}=0$ and $\varpi_{i}=\rho_{i}+k l_{i}$. Hence, the divergence of the stress tensor when evaluated using an induced rigged connection is given simply by

$$
(\operatorname{div} T)_{j}=D_{i} T_{j}^{i}
$$

The final step is to relate this divergence to the constraint equations on $\mathcal{N}$. The contracted Codazzi equation of the null surface is given by equation (6.3) reference [52],

\footnotetext{
${ }^{10}$ Such a generalized divergence can be defined for any tensor $A^{i}{ }_{j}$ for which $n^{j}$ is an eigenvector, $A^{i}{ }_{j} n^{j}=$ $\alpha n^{i}$, and whose spatial component is symmetric, $A_{j}^{i} q_{i k}=A_{k}^{i} q_{i j}$. Any such tensor can be decomposed as $A^{i}{ }_{j}=2 a^{i k} q_{k j}-n^{i} a_{j}$ with $a^{i j}$ symmetric, and the pair $\left(a^{i j}, a_{j}\right)$ are only determined up to shifts of the form $a^{i j} \rightarrow a^{i j}+V^{i} n^{j}+n^{i} V^{j}, a_{j} \rightarrow a_{j}+q_{j k} V^{k}$. Defining the divergence by

$$
(\operatorname{div} A)_{j} \xi^{j} \eta=d\left(A^{i}{ }_{j} \xi^{j} \eta_{i}\right)-\eta\left(a^{i j} £_{\xi} q_{i j}+a_{i} £_{\xi} n^{i}\right),
$$

one can check that the resulting expression is insensitive to the ambiguity in the definition of $a^{i j}$ and $a_{i}$.
} 
which evaluates to

$$
\begin{aligned}
\Pi_{c}^{b} R_{a b} n^{a} & =\Pi_{c}^{b}\left[\nabla_{a} K_{b}^{a}+n^{a} \nabla_{a} \rho_{b}+(k+\Theta) \rho_{b}-\nabla_{b}(k+\Theta)-K_{b c} c^{a} \nabla_{a} n^{c}\right] \\
& =D_{a} K_{c}^{a}+n^{a} D_{a} \rho_{c}+W \rho_{c}-D_{c} W \\
& =D_{a} W_{c}^{a}-D_{c} W
\end{aligned}
$$

where to get to the second line we used $\nabla_{a} K_{b}^{a}=\Pi_{d}^{a} \nabla_{a} K_{b}^{d}-n_{a} l^{c} \nabla_{c} K_{b}^{a}=\Pi_{d}^{a} \nabla_{a} K_{b}^{d}+$ $l^{c} \nabla_{c} n^{a} K_{a b}$, and for the third line we used $D_{a} n^{a}=W$. We immediately recognize this to be proportional to the divergence of the null Brown-York stress tensor, and hence we conclude that this divergence vanishes on shell,

$$
D_{i} T_{j}^{i}=-8 \pi G \Pi_{j}^{b} R_{a b} n^{a}=0 .
$$

Often, the null Codazzi equations are separated into the $n^{j}$ component, which is just the Raychaudhuri equation, and a spatial component, which is known as the Damour-NavierStokes equation $[52,53]$.

As in the timelike case, the vanishing of $(\operatorname{div} T)_{j}$ allows us to now conclude the equality of the Wald-Zoupas and Brown-York charge densities. The Wald-Zoupas charge densities associated with a Dirichlet flux condition are again given by the general formula (2.10) using the expressions (3.23) and (3.28) for $\ell$ and $\beta$. They satisfy the continuity equation $d h_{\xi}=\mathcal{E}_{\xi}$ [18], which, according to (3.29), is equal to $d j_{\xi}$ after imposing the stress tensor conservation equation $f_{\xi}=0$. We can therefore conclude the equality of $h_{\xi}$ and $j_{\xi}$, subject to the same caveats described above equation (2.14) regarding the addition of exact forms $d c_{\xi}$ to each. This then demonstrates that for symmetries that act covariantly on $q_{i j}$ and $n^{i}$,

$$
H_{\xi}=\int_{\mathcal{S}} h_{\xi}=\int_{\mathcal{S}} j_{\xi}=\mathcal{Q}_{\xi}^{\mathrm{BY}}
$$

The on-shell continuity equation $d h_{\xi}=d j_{\xi}=\mathcal{E}_{\xi}$ implies that the Brown-York charges (3.42) are not conserved between cuts of the boundary $\mathcal{N}$, but instead satisfy a flux-balance equation in which the difference of the charges is given by the integral of $\mathcal{E}_{\xi}$ between the cuts $[18,31]$.

When utilizing additional intrinsic boundary terms during holographic renormalization procedures for asymptotic charges, the two notions of charges will continue to agree, provided the boundary counterterms $\ell_{\text {ct }}$ are fully covariant with respect to the diffeomorphisms from which the charges are being constructed.

\subsection{Comparison to other null Brown-York stress tensors}

While the expression (3.35) for the Brown-York stress tensor on a null surface is a novel result of the present work, the idea of applying the Brown-York construction to null surfaces has been considered previously, see e.g. [45, 46, 72, 73]. Of particular note are the works of Jafari [45] and Donnay and Marteau [46], which both offer proposals for a full stress tensor associated with a null surface. These proposals each differ slightly from the stress tensor (3.35) due to different choices in boundary conditions and intrinsic structures on the null surface when defining the subregion variational principle, and in this section we briefly 
describe the difference between these various proposals for the null Brown-York stress tensor. In making comparisons, we will refer to the stress tensor (3.35) of the present work as the normal Brown-York stress tensor, since it arises from a variational principle that fixes the null normal $n_{a}$ at $\mathcal{N}$.

The stress tensor defined by Jafari [45] bears many similarities to (3.35), as both are derived from a Dirichlet variational principle. The main difference is that Jafari does not impose the boundary condition that the surface $\mathcal{N}$ remain null for all variations of the metric, and the variations that change the null character of the surface are related to the energy density computed by the stress tensor. Additionally, Jafari employs a foliation by codimension-2 surfaces in the region of spacetime near $\mathcal{N}$, following the constructions in refs. $[42,44,74]$, which induces a preferred foliation of the null hypersurface. This has the effect of allowing for some variations that rescale the null generator, $\delta n_{a} \propto n_{a}$, in contrast to the boundary condition $\delta n_{a}=0$ employed in the present work. A final difference is that Jafari obtains a stress tensor with covariant indices $T_{i j}$, making the comparison to the mixed index version $T_{j}^{i}$ somewhat subtle. However, because the null surface in Jafari's construction comes equipped with a preferred auxiliary null vector $l^{a}$ due to the local foliation by codimension-2 surfaces, a prescription can be given to define an equivalent mixed-index tensor. This amounts to defining an intrinsic Lorentzian metric on the null surface using the induced auxiliary one-form $l_{i}$ via

$$
h_{i j}=-l_{i} l_{j}+q_{i j},
$$

whose inverse is given by $h^{i j}=-n^{i} n^{j}+q^{i j}$. This choice then implies that lowering the index of the null generator $n^{i}$ yields the auxiliary one-form, $n^{i} h_{i j}=l_{j}$. When computing the components of the stress tensor, this prescription can be implemented through the relation $T_{j}^{i} l_{i}=T_{i j} n^{i}$.

The most straightforward way to compare to Jafari's expressions is then to decompose the normal Brown-York stress tensor (3.35) into an energy density $E$, momentum $P_{i}$, and a spatial stress tensor $\Sigma_{j}^{i}$ according to

$$
\begin{aligned}
E & =-T_{j}^{i} n^{j} l_{i}=\frac{\Theta}{8 \pi G} \\
P_{k} & =T_{j}^{i} l_{i} q^{j}{ }_{k}=\frac{\varpi_{k}}{8 \pi G} \\
\Sigma_{j}^{i} & =T_{l}^{k} q_{j}^{l} q_{k}^{i}=-\frac{1}{8 \pi G}\left(K_{j}^{i}-(\Theta+k) q_{j}^{i}\right) .
\end{aligned}
$$

The expressions for the momentum density $P_{j}$ and the spatial stress tensor $\Sigma_{j}^{i}$ coincide with Jafari's expressions in equations (37) and (38) of ref. [45]; however, the energy density (3.44) differs from Jafari's expression, which instead involves the expansion of the codimension-2 foliation along the auxiliary null direction $l^{a}$ transverse to the surface. The difference in the expression for the energy density is entirely due to the different choice of background structures and boundary conditions Jafari employs in defining the null surface variational principle.

The stress tensor of Donnay and Marteau [46] is obtained by a somewhat different procedure. Rather than working directly on the null surface, they consider a sequence of time- 
like surfaces that limit to the null surface. On each timelike surface, the usual Brown-York stress tensor can be constructed from the extrinsic curvature $K^{i}{ }_{j}$ as in (2.7). Although this diverges as the null limit is taken, the densitized stress tensor $T_{j}^{i} \eta_{i}$ has a finite limit, which defines a tensor $\left(T_{\mathrm{NL}}\right)^{i}{ }_{j}$ on $\mathcal{N}$ that we refer to as the null-limit Brown-York stress tensor.

To see how this works in more detail, it is helpful to consider an unnormalized normal vector to the timelike hypersurfaces, which smoothly limits to the null normal $n_{a}$ of the null surface. Hence, we consider a function $\Phi$ whose level sets foliate the region near $\mathcal{N}$, such that the unnormalized normal $n_{a}=\nabla_{a} \Phi$ limits to the null normal at $\Phi=0$. The norm of $n_{a}$ vanishes as the null surface is approached, and hence there must be a function $\kappa$ which we refer to as the surface gravity, that satisfies the equation

$$
g^{a b} n_{a} n_{b}=2 \kappa \Phi,
$$

and generically $\kappa$ has a nonzero limit to $\mathcal{N} .{ }^{11}$ In terms of $n_{a}$, the projector onto the timelike surfaces can be expressed as

$$
h^{a}{ }_{b}=\delta^{a}{ }_{b}-\frac{n^{a} n_{b}}{2 \kappa \Phi} .
$$

Using this projector, we can construct a shape operator $\tilde{K}_{b}^{a}$ from the covariant derivative of the normal by the relation

$$
\tilde{K}_{b}^{a}=h^{a}{ }_{c} h_{b}^{d} \nabla_{d} n^{c}=h_{b}^{d}\left[\nabla_{d} n^{a}-\frac{1}{2} n^{a} \nabla_{d} \log \kappa\right]
$$

where in obtaining the final expression on the right hand side of (3.49), we used the fact that $h_{b}^{a}{ }_{b} \nabla_{a} \Phi=h_{b}^{a} n_{a}=0$. By definition, this tensor is tangential to the constant $\Phi$ surfaces on its contravariant index, and hence pulls back to a well defined tensor $\tilde{K}_{j}{ }_{j}$ on the surface, whose defining relation is

$$
\tilde{K}_{j}^{i} \Pi_{i}^{a}=\Pi^{b}{ }_{j}\left[\nabla_{b} n^{a}-\frac{1}{2} n^{a} \nabla_{b} \log \kappa\right]
$$

where $\Pi^{a}{ }_{i}$ is the pullback map to each constant $\Phi$ surface. It is related to the more familiar extrinsic curvature of the surface $K^{i}$, which is constructed from the covariant derivative of the unit normal $\hat{n}^{a}=\frac{n^{a}}{\sqrt{2 \kappa \Phi}}$ by a simple rescaling [47],

$$
K_{j}^{i}=\frac{1}{\sqrt{2 \kappa \Phi}} \tilde{K}_{j}^{i}
$$

The terms appearing in (3.50) have manifestly finite limits to $\mathcal{N}$, and we see that $\tilde{K}_{j}{ }_{j}$ limits to a shifted version of the null surface shape operator,

$$
\tilde{K}_{j}^{i} \stackrel{\Phi \rightarrow 0}{\longrightarrow} W_{j}^{i}-n^{i} a_{j}
$$

where

$$
a_{j}=\frac{1}{2} D_{j} \log \kappa
$$

is the acceleration of the normal vector of the timelike foliation.

\footnotetext{
${ }^{11}$ For generic null surfaces, the surface gravity $\kappa$ defined by equation (3.47) can differ from the inaffinity $k$ defined by equation (3.6), although these two definitions agree for Killing horizons, as well as whenever $n_{a}$ is chosen to be a pure gradient.
} 
Since $\tilde{K}_{j}{ }_{j}$ has a finite limit, it is clear from equation (3.51) that the extrinsic curvature tensor $K^{i}{ }_{j}$ diverges as $\Phi^{-\frac{1}{2}}$ in the null limit. The Brown-York tensor on the timelike surfaces will then similarly diverge in the null limit. However, as discussed at the beginning of section 3, a more natural object to consider is the densitized stress tensor $T_{j}^{i} \hat{\eta}_{i}$, where $\hat{\eta}$ is the induced volume form. Since $\hat{\eta}$ vanishes as $\sqrt{2 \kappa \Phi}$ as the surface becomes null, we see that the densitized stress tensor has a finite null limit. We can then turn this into a tensor on the null surface using the volume form $\eta$ associated with the null normal $n_{a}$ to obtain the null-limit Brown-York stress tensor,

$$
\left(T_{\mathrm{NL}}\right)_{j}^{i}=-\frac{1}{8 \pi G}\left(\tilde{K}^{i}{ }_{j}-\tilde{K} \delta^{i}{ }_{j}\right)=-\frac{1}{8 \pi G}\left(W^{i}{ }_{j}-W \delta^{i}{ }_{j}-n^{i} a_{j}+\left(n^{k} a_{k}\right) \delta_{j}^{i}\right) .
$$

This final expression is the stress tensor obtained by Donnay and Marteau [46], which differs from the normal Brown-York stress tensor (3.35) by the acceleration terms $a_{j}$ which involve gradients of the surface gravity,

$$
\left(T_{\mathrm{NL}}\right)^{i}{ }_{j}=T_{j}^{i}+\frac{1}{8 \pi G}\left(n^{i} a_{j}-n^{k} a_{k} \delta_{j}^{i}\right) .
$$

An interesting feature is that these corrections cancel out of the energy density, which is still given by (3.44) when using the null-limit stress tensor. Gradients of $\kappa$ then enter into expressions for the momentum $P_{k}$ and the spatial stress tensor $\Sigma_{j}^{i}$.

Finally, we mention that the conservation equation for the null-limit stress tensor is somewhat different from the conservation equation (3.41) for the normal Brown-York stress tensor (3.35). Continuing to employ the induced rigged connection defined in 3.15 and using that $D_{i}\left(W^{i}{ }_{j}-W \delta^{i}{ }_{j}\right)=0$ on shell and $d a=0$, we find that

$$
\begin{aligned}
D_{i}\left(T_{\mathrm{NL}}\right)^{i}{ }_{j} & =\frac{1}{8 \pi G} D_{i}\left(n^{i} a_{j}-\delta^{i}{ }_{j}\left(n^{k} a_{k}\right)\right) \\
& =-\frac{1}{8 \pi G}\left(W^{i}{ }_{j}-W \delta^{i}{ }_{j}\right) a_{i} \\
& =\left(T_{\mathrm{NL}}\right)^{i}{ }_{j} a_{i}
\end{aligned}
$$

where the last line uses $a_{i}\left(n^{i} a_{j}-\delta^{i}{ }_{j} n^{k} a_{k}\right)=0$. This correction to the conservation equation involving the acceleration is expected from the perspective of the null limit, since $\left(T_{\mathrm{NL}}\right)^{i}{ }_{j}$ arises as the limit of rescaled version of the timelike Brown-York stress tensor,

$$
\left(T_{\mathrm{NL}}\right)_{j}^{i}=\lim _{\Phi \rightarrow 0}\left[-\frac{\sqrt{2 \kappa \Phi}}{8 \pi G}\left(K^{i}{ }_{j}-K \delta^{i}{ }_{j}\right)\right] .
$$

Since the usual Brown-York stress tensor constructed from $K_{j}^{i}$ is covariantly conserved onshell with respect to the induced connection on the timelike surfaces, the rescaled stress tensor satisfies a modified conservation equation exactly of the form (3.56). It would be interesting to explore in more detail these modifications of the conservation equation, and their relation to the Carrollian conservation equations described in [46, 69-71]. In particular, there may be a different notion of connection on the null surface that is naturally induced from the null limit that makes the interpretation of the conservation more straightforward. 


\section{Anomalous transformations}

The previous sections demonstrated that the Brown-York and canonical Wald-Zoupas charges agree, provided that the diffeomorphisms being considered act covariantly on the intrinsic boundary structures and on the boundary term $\ell$ in the action. In some contexts, however, it is useful to consider charges for transformations that act anomalously on these structures. For example, the appearance of central extensions in the algebra of Virasoro charges on black hole horizons [16, 17] was shown in [18] to be a consequence of such anomalous transformations. They arise in situations where additional background structure is introduced when constructing the subregion action that was not originally present in the definition of the field configuration space. Transformations which do not preserve the background structure will act anomalously on functionals that depend on it.

In this section, we investigate whether the two notions of charges continue to agree when considering these more general, anomalous transformations. For finite timelike surfaces, all diffeomorphisms that are tangent to the surface act covariantly on the GHY boundary term (2.3) and the intrinsic metric $h_{i j}$, and hence the equality between BrownYork and canonical charges holds. By contrast, it was shown in [18] that certain diffeomorphisms of a null surface can act anomalously on the boundary term (3.23) and intrinsic quantities. For this reason, we restrict attention to null surfaces in this section. In principle, anomalous transformations can also arise for timelike surfaces if the diffeomorphism contains a nonzero transverse component. This situation is particularly relevant for asymptotic symmetries of anti-de Sitter space, where such transformations give rise to the well-known holographic Weyl anomalies of the dual CFT [12, 32, 34, 57, 62]. Although we do not analyze this case in detail due to subtleties in handling transverse surface deformations, we expect similar reasoning to apply in this case as well. It would be interesting to explore this case in more detail in the future.

As discussed in section 3.2, since the Dirichlet variational principle with a null boundary is formulated by fixing a preferred null normal $n_{a}$, anomalies can arise from diffeomorphisms which are tangent to $\mathcal{N}$ but only preserve $n_{a}$ up to a rescaling. This rescaling $w_{\xi}$ is known as the boost weight of the transformation, and is defined through the equation

$$
£_{\xi} n_{a}=w_{\xi} n_{a} .
$$

On the other hand, since $n_{a}$ is taken to be a fixed quantity, it cannot in particular transform nontrivially under diffeomorphisms, so that $\delta_{\xi} n_{a}=0$. It is convenient to parameterize the failure of $n_{a}$ to transform covariantly through an anomaly operator $\Delta_{\xi}$ [74], defined by

$$
\delta_{\xi} n_{a}=£_{\xi} n_{a}+\Delta_{\xi} n_{a}
$$

By the above discussion, we immediately find $\Delta_{\xi} n_{a}=-w_{\xi} n_{a}$, and this equation also fixes the anomalous transformation of the intrinsic null generator,

$$
\Delta_{\xi} n^{i}=-w_{\xi} n^{i}
$$


Such transformations also act anomalously on the null boundary term (3.23), and this anomaly was computed in [18] to be

$$
\Delta_{\xi} \ell=\frac{\left(n^{a} \nabla_{a} w_{\xi}\right) \eta}{8 \pi G}
$$

Since the degenerate induced metric is independent of the normalization of $n_{a}$, it continues to transform covariantly, $\Delta_{\xi} q_{i j}=0$.

Note that the conservation equation (3.41) for the null Brown-York stress tensor is independent of the vector field $\xi^{a}$ and whether or not it acts anomalously. Because of this, once the gravitational constraint equations are imposed, the divergence of the Brown-York charge density always satisfies

$$
d j_{\xi}=\mathcal{E}_{\xi},
$$

with $\mathcal{E}_{\xi}$ given in (3.29). On the other hand, the canonical charge density $h_{\xi}$ satisfies a modified continuity equation derived in [18],

$$
\begin{aligned}
d h_{\xi} & =-\pi^{i j} £_{\xi} q_{i j}-\pi_{i} £_{\xi} n^{i}-\pi_{i} \Delta_{\hat{\xi}} n^{i}-\Delta_{\hat{\xi}} \ell \\
& =d j_{\xi}+(\operatorname{div} T)_{j} \xi^{j} \eta-\frac{\eta}{8 \pi G}\left(\Theta w_{\xi}+n^{i} D_{i} w_{\xi}\right) .
\end{aligned}
$$

The final term in (4.7) reduces to the exact term $-\frac{1}{8 \pi G} d\left(w_{\xi} \mu\right)$ on the null surface. We can therefore conclude that on shell, the Brown-York and Wald-Zoupas charge densities are related via

$$
h_{\xi}=j_{\xi}-\frac{1}{8 \pi G} w_{\xi} \mu,
$$

and therefore the charges differ by the integral of the anomaly over the cut $\mathcal{S}$,

$$
H_{\xi}=\mathcal{Q}_{\xi}^{\mathrm{BY}}-\frac{1}{8 \pi G} \int_{\mathcal{S}} w_{\xi} \mu
$$

As an explicit example of how such a difference between Brown-York and Wald-Zoupas charges can arise, we now consider the symmetries and charges at a finite null boundary found by Chandrasekaran, Flanagan, and Prabhu (CFP) [50]. ${ }^{12}$ In this example, the field configuration space for general relativity in a subregion with a null boundary is further restricted to fix $n^{i}$ and $k$ on the null surface. It was shown that the symmetry group of this field configuration space is

$$
\operatorname{diff}(\mathcal{S}) \ltimes \mathfrak{s},
$$

where $\mathcal{S}$ is the base space of $\mathcal{N}$, viewing $\mathcal{N}$ as a fiber bundle where the null generators are fibered over $\mathcal{S}$, and $\mathfrak{s}$ consists of the set of vector fields $\xi^{a}=f n^{a}$ for functions $f: \mathcal{S} \rightarrow \mathbb{R}$ which satisfy

$$
£_{n}\left(£_{n}+\kappa\right) f=0 .
$$

The solutions correspond to angle-dependent translations and angle-dependent rescalings of the integral curves of the null generator. The $\operatorname{diff}(\mathcal{S})$ generators are represented by vector fields $\xi^{a}=X^{a}$ where $X^{a} n_{a}=X^{a} l_{a}=0$. The Wald-Zoupas charges associated to

\footnotetext{
${ }^{12}$ The same algebra was recently considered as an extended symmetry algebra of null infinity in ref. [10].
} 
this symmetry group were computed in CFP using covariant phase space methods, with the result that on any cross-section $S$ of the null boundary, ${ }^{13}$

$$
H_{\xi}=-\frac{1}{8 \pi G} \int_{\mathcal{S}} \mu\left(\Theta f-£_{n} f+W_{j}^{i} X^{j} l_{i}-l_{i} £_{X} n^{i}\right)
$$

where we have shifted the boundary term used in CFP by $\ell \rightarrow \ell-\frac{1}{8 \pi G}(k-\Theta) \eta$ in order to agree with the choice of boundary term and total derivative term $\beta$ in the present work. We now compare these charges to the ones which result from the Brown-York stress tensor (3.35).

The Brown-York charges for these symmetries are

$$
\mathcal{Q}_{\xi}^{\mathrm{BY}}=-\int_{\mathcal{S}} T^{i}{ }_{j} \xi^{j} \eta_{i}=-\frac{1}{8 \pi G} \int_{\mathcal{S}} \mu\left(\Theta f+W^{i}{ }_{j} X^{j} l_{i}\right)
$$

which differs from the Wald-Zoupas charges. However, note that the field space defined in CFP contains an anomalous transformation of $n^{i}$ (4.3). A simple computation of $w_{\xi}$ results in

$$
w_{\xi}=-£_{n} f-l_{i} £_{X} n^{i} .
$$

Therefore, the Wald-Zoupas and Brown-York charges differ by the term

$$
H_{\xi}-\mathcal{Q}_{\xi}^{\mathrm{BY}}=-\frac{1}{8 \pi G} \int_{\mathcal{S}} \mu w_{\xi},
$$

in agreement with the general relation (4.9).

We further remark on an important difference between the Wald-Zoupas and BrownYork charges. Choose coordinates $\left(u, x^{A}\right)$ on $\mathcal{N}$, where $u$ is an affine parameter for the null generator, i.e. $n^{i}=\partial_{u}, k=0$, and $x^{A}$ are coordinates for $\mathcal{S}$. Furthermore, we can always choose $u$ such that $\mathcal{S}$ is at $u=0$. The condition (4.11) can be solved to get $f\left(u, x^{A}\right)=f_{0}\left(x^{A}\right)+u f_{1}\left(x^{A}\right)$. The angle-dependent translations, $f_{0}\left(x^{A}\right)$, contribute to both (4.12) and (4.13), but the angle-dependent rescalings $f_{1}\left(x^{A}\right)$ only contribute to (4.12). In particular, they only contribute through the anomaly term. Ultimately this is because the rescaling generators come from boost generators in the bulk spacetime, which vanish at $\mathcal{S}$ but have non-vanishing first derivative there. While the Wald-Zoupas charges depend on first derivatives of the symmetry generators, the Brown-York charges do not. ${ }^{14}$ Note that if we take $\mathcal{N}$ to be the Killing horizon of a stationary black hole, then the Wald entropy [75] arises purely from the anomaly.

\section{Discussion and future work}

This paper has presented a novel expression for a Brown-York stress tensor associated with null hypersurfaces in general relativity and has established its two key features: covariant

\footnotetext{
${ }^{13}$ In CFP the Wald-Zoupas charges were found using the stationarity condition that the flux should vanish for all perturbations on a solution for which $\mathcal{N}$ is stationary. For the field space considered in CFP, which imposes boundary conditions fixing $n^{i}$ and $k$, this happens to agree with the Dirichlet variational principle adopted in the present paper.

${ }^{14}$ Of course, if we evaluate the Brown-York charges for the rescaling symmetries on any other cross-section, after having fixed $\mathcal{S}$ to lie at $u=0$, then it will be non-vanishing.
} 
conservation with respect to an induced connection on the null surface, and the relationship between the associated Brown-York charges and canonical charges obtained via the Wald-Zoupas construction. The conservation equation was shown to be equivalent to the gravitational constraint equations on the null surface, consisting of the Raychaudhuri and Damour-Navier-Stokes equations. The latter name refers to the analogy made by Damour between this evolution equation for kinematical quantities on a black hole horizon and the Navier-Stokes equation of hydrodynamics. Reformulating it as a conservation equation for a stress tensor sheds light on the reason for this analogy: at their core, hydrodynamical equations are simply conservation equations for a fluid stress tensor. The striking feature of the conservation equation (3.41) for the null Brown-York stress tensor (3.35) is that it explicitly takes the form of a covariant divergence of stress tensor with respect to a well-defined connection $D_{i}$ on the null surface. This is in contrast to previous works $[46,53]$, which generally separate these conservation equations into components, somewhat obscuring their interpretation. It is also in contrast to formulations which express the conservation equations as flux balance equations such as in [74], which are more analogous to the on-shell relation $d j_{\xi}=\mathcal{E}_{\xi}$ as in equation (3.30). While imposing the flux balance equation for every choice of $\xi^{i}$ implies the conservation equation and vice-versa, the conservation equation (3.41) is more directly related to the dynamics of the system and the hydrodynamical equations of motion.

Note that the connection between gravitational equations and hydrodynamics features prominently in the fluid-gravity correspondence in holography $[76,77]$. We therefore expect the identification of the null Brown-York stress tensor and its conservation equation to yield important insights into holographic correspondences involving null boundaries, including celestial holography and for holographic descriptions of subregions bounded by null surfaces. Some ideas in this direction have been explored in [68, 71, 78-80]. It would also be interesting to relate this Brown-York stress tensor with the stress tensor of celestial conformal field theory [81] to see if there exists an equivalence between the two, analogous to that in AdS/CFT. This would be an important step in establishing the holographic dictionary between general relativity in asymptotically flat spacetimes and celestial conformal field theory.

An immediate future direction would be to carry out this analysis at future null infinity in four-dimensional asymptotically flat spacetimes to compare the Brown-York charges with the charges obtained, for example, in $[8,55,82]$ for the symmetries corresponding to the (generalized) Bondi-Metzner-Sachs (BMS) algebra. For ordinary BMS symmetries in Bondi coordinates, it has been demonstrated in [72] that the Brown-York charges reproduce the expression for BMS charges when realizing null infinity as a null limit of timelike surfaces. More generally, however, applying the Brown-York construction to asymptotic boundaries requires additional boundary counterterms $\ell^{\text {ct }}$ in the subregion action to yield a finite renormalized on-shell action functional $S^{\mathrm{cl}}$. These counterterms must be suitably covariant to preserve the conservation equation of the stress tensor and to yield agreement with the canonical charges, and it is an interesting question whether a fully covariant renormalized action can be obtained. There are also choices in how one foliates spacetime near null infinity, and for some choices of foliation, the null-limit stress tensor of Donnay and Marteau [46] may be a more appropriate object to consider, especially when looking 
for covariant counterterms. It would further be interesting to determine how the continuity equation for the Brown-York charges relates to the flux-balance equations for generalized BMS charges explored in [83]; given that both are consequences of the Einstein equation, establishing a relationship should be possible.

Although we focused on vacuum general relativity in this work, generalizations involving the inclusion of matter or modified gravitational theories are possible. As long as the modified action admits a Dirichlet variational principle, the general construction of the boundary stress tensor follows. With matter fields, the on-shell action will also depend on the boundary values of the fields, and there will be contributions to the stress tensor coming from the matter action. Similarly, higher curvature theories such as Lovelock gravity will also yield a well-defined boundary stress tensor utilizing modified expressions for $\pi^{i j}$ and $\pi_{i}$ on the null surface after adding the appropriate null boundary terms for these theories [84].

\section{Acknowledgments}

We thank Luca Ciambelli, Daniel Harlow, Rob Leigh, and Don Marolf for helpful discussions. We also thank an anonymous referee for comments that improved the presentation of this work. E.F. and I.S. are supported in part by NSF grants PHY-1707800 and PHY-2110463. I.S. also acknowledges support from the John and David Boochever prize fellowship in fundamental theoretical physics. AJS is supported by the Air Force Office of Scientific Research under award number FA9550-19-1-036. Research at Perimeter Institute is supported in part by the Government of Canada through the Department of Innovation, Science and Economic Development and by the Province of Ontario through the Ministry of Colleges and Universities. V.C. is supported in part by the Berkeley Center for Theoretical Physics; by the Department of Energy, Office of Science, Office of High Energy Physics under QuantISED Award DE-SC0019380 and under contract DEAC02-05CH11231; by the National Science Foundation under grant PHY-1820912; and by a grant from the Simons Foundation (816048, VC).

\section{A Connections for Carroll geometries}

In this appendix, we derive the compatibility relations for a class of connections associated with a Carroll geometry arising from a spacetime rigging vector. As explained in section 3.1, the first step in defining an affine connection associated with a Carroll geometry $\left(q_{i j}, n^{i}\right)$ is to choose an Ehresmann connection, defined in terms of a one-form $l_{i}$, whose only requirement is that $l_{i} n^{i}=-1$. This connection allows one to specify a class of horizontal vectors, consisting of any vector satisfying $V^{i} l_{i}=0$. The choice of $l_{i}$ already determines a set of differential invariants coming from the exterior derivative $d l$ that are defined independently of any choice of affine connection. In general, this exterior derivative can be decomposed as

$$
d l=b \wedge l+r
$$


where $b_{i}$ and $r_{i j}$ are both horizontal differential forms. We refer to $b_{i}$ as the boost form and $r_{i j}$ as the curvature of the Ehresmann connection. ${ }^{15}$

We wish to show that a connection $D_{i}$ on a Carroll manifold induced as a rigged connection associated with an embedded null surface in spacetime implies the relations (3.17)(3.20). Additionally, we will derive the expression for $D_{i} l_{j}$ arising from such a connection. Equation (3.18) follows immediately from the definitions of the induced connection and the shape operator, (3.15) and (3.4). To derive (3.17), we note that $q_{i j}$ maps via the inclusion $\Pi_{a}^{i}$ to a spacetime tensor $q_{a b}$ that annihilates both $n^{a}$ and $l^{a}$, given by

$$
q_{a b}=g_{a b}+l_{a} n_{b}+n_{a} l_{b}
$$

Then we can compute

$$
\begin{aligned}
D_{i} q_{j k} & =\Pi^{a}{ }_{i} \Pi^{b}{ }_{j} \Pi^{c}{ }_{k}\left(\nabla_{a}\left(g_{b c}+l_{b} n_{c}+n_{b} l_{c}\right)\right) \\
& =\Pi^{a}{ }_{i} \Pi^{b}{ }_{j} \Pi^{c}{ }_{k}\left(l_{b} \nabla_{a} n_{c}+l_{c} \nabla_{a} n_{b}\right) \\
& =l_{j} K_{i k}+l_{k} K_{i j} .
\end{aligned}
$$

For the relation (3.19), we note that the inclusion map sends $\eta$ defined intrinsically on the null surface $\mathcal{N}$ to a spacetime $(d-1)$-form $\eta_{a \ldots}$ that is related to the spacetime volume form by the equation $\epsilon=n \wedge \eta$. Furthermore $\eta_{a \ldots}$ must satisfy $l^{a} \eta_{a \ldots}=0$, and hence it can be defined by the equation $\eta=-i_{l} \epsilon$. Then we have

$$
\begin{aligned}
D_{i} \eta_{j \ldots} & =-\Pi_{i}^{a} \Pi^{b}{ }_{j} \cdots \nabla_{a} l^{c} \epsilon_{c b \ldots} \\
& =-\Pi^{a}{ }_{i} \Pi^{b}{ }_{j} \cdots \nabla_{a} l^{c}\left(n_{c} \eta_{b \ldots}-(d-1) \eta_{c[\ldots} n_{b]}\right) \\
& =-\rho_{i} \eta_{j \ldots} .
\end{aligned}
$$

Then using that $\mu=i_{n} \eta$ in $\mathcal{N}$, relations (3.18) and (3.19) fix the expression for $D_{i} \mu$ :

$$
D_{i} \mu=D_{i}\left(n^{j} \eta_{j}\right)=W_{i}^{j} \eta_{j}-n^{j} \rho_{i} \eta_{j}=K_{i}^{j} \eta_{j}
$$

Finally, we should examine the expression for $D_{i} l_{j}$. Since $D_{i}$ is torsionless, the antisymmetric components are fixed in terms of $b_{i}$ and $r_{i j}$ in terms of (A.1). We can also compute the components parallel to $l_{i}$ by the relations

$$
\begin{aligned}
n^{j} D_{i} l_{j} & =\Pi^{a}{ }_{i} n^{b} \nabla_{a} l_{b}=\rho_{i} \\
n^{i} D_{i} l_{j} & =\Pi^{b}{ }_{j} n^{a} \nabla_{a} l_{b}=\Pi^{b}{ }_{j}\left(n^{a}(d l)_{a b}+n^{a} \nabla_{b} l_{a}\right)=b_{j}+\rho_{j} .
\end{aligned}
$$

The remaining components of $D_{i} l_{j}$ are purely horizontal, and the antisymmetric horizontal component is simply $r_{i j}$ according to equation (A.1). The remaining horizontal symmetric component is an independent tensor $\nu_{i j}$ characterizing the extrinsic geometry of the null surface. Hence, using $\rho_{i}=-k l_{i}+\varpi_{i}$, we arrive at the final decomposition of $D_{i} l_{j}$,

$$
D_{i} l_{j}=k l_{i} l_{j}-\varpi_{i} l_{j}-l_{i} \varpi_{j}-l_{i} b_{j}+\frac{1}{2} r_{i j}+\nu_{i j} .
$$

\footnotetext{
${ }^{15}$ In [66], the quantity $b_{i}$ was referred to as the "acceleration" and $r_{i j}$ as the "Carrollian torsion."
} 
From the intrinsic perspective, we can ask to what extent these relations fully specify an affine connection that is in a certain sense compatible with the Carroll structure. According to the above relations, we see that the additional data needed to fix a torsionless connection on the intrinsic Carroll geometry is an Ehresmann connection $l_{i}$, a rotation one-form $\rho_{i}$, and a horizontal symmetric tensor $\nu_{i j}$. Choosing a coordinate system $\left(u, x^{A}\right)$ in which $n^{i}=\partial_{u}^{i}$ and $x^{A}$ are coordinates for the horizontal directions, a generic Ehresmann connection can be parameterized by $l_{i}=-\nabla_{i} u+w_{B} \nabla_{i} x^{B}$. Then, defining the connection coefficients in this coordinate system according to $D_{i} V^{j}=\partial_{i} V^{j}+\gamma_{j k}^{i} V^{k}$, one can then check that equations (3.17), (3.18), and (A.8) fully fix all components of $\gamma_{j k}^{i}$, and hence these equations uniquely specify the connection $D_{i}$.

Note that much of the work on connections associated with Carrollian structures has focused on connections that preserve $q_{i j}$ and $n^{i}[65,66,68]$. When $K_{i j}$ is nonvanishing, these connections necessarily must involve torsion [85]; for example, torsion is necessary in order to be compatible with the equation $K_{i j}=\frac{1}{2} £_{n} q_{i j}$. In the present work, however, we found that the natural connection that arises in considerations of the null Brown-York stress tensor and conservation equations involves a torsionless connection that generically does not preserve $q_{i j}$ and $n^{i}$. Such connections do not appear to have been considered in recent works on Carrollian geometry, and it would be interesting to further investigate the geometric properties of these connections in the future. A related set of connections satisfying equation (3.17) but not (3.18) have been examined previously in [51], and general properties of affine connections in Carrollian geometries have been explored in [86].

Open Access. This article is distributed under the terms of the Creative Commons Attribution License (CC-BY 4.0), which permits any use, distribution and reproduction in any medium, provided the original author(s) and source are credited.

\section{References}

[1] G. Barnich and C. Troessaert, Aspects of the BMS/CFT correspondence, JHEP 05 (2010) 062 [arXiv: 1001.1541] [INSPIRE].

[2] G. Barnich and C. Troessaert, BMS charge algebra, JHEP 12 (2011) 105 [arXiv:1106.0213] [INSPIRE].

[3] A. Strominger, On BMS invariance of gravitational scattering, JHEP 07 (2014) 152 [arXiv:1312.2229] [INSPIRE].

[4] T. He, V. Lysov, P. Mitra and A. Strominger, BMS supertranslations and Weinberg's soft graviton theorem, JHEP 05 (2015) 151 [arXiv: 1401.7026] [INSPIRE].

[5] A. Strominger, Lectures on the infrared structure of gravity and gauge theory, arXiv: 1703.05448 [INSPIRE].

[6] M. Campiglia and A. Laddha, Asymptotic symmetries and subleading soft graviton theorem, Phys. Rev. D 90 (2014) 124028 [arXiv:1408.2228] [InSPIRE].

[7] M. Campiglia and A. Laddha, New symmetries for the gravitational S-matrix, JHEP 04 (2015) 076 [arXiv : 1502.02318] [inSPIRE]. 
[8] G. Compère, A. Fiorucci and R. Ruzziconi, Superboost transitions, refraction memory and super-Lorentz charge algebra, JHEP 11 (2018) 200 [Erratum ibid. 04 (2020) 172] [arXiv: 1810.00377] [INSPIRE].

[9] E.E. Flanagan, K. Prabhu and I. Shehzad, Extensions of the asymptotic symmetry algebra of general relativity, JHEP 01 (2020) 002 [arXiv: 1910.04557] [INSPIRE].

[10] L. Freidel, R. Oliveri, D. Pranzetti and S. Speziale, The Weyl BMS group and Einstein's equations, JHEP 07 (2021) 170 [arXiv:2104.05793] [INSPIRE].

[11] S. Hollands, A. Ishibashi and D. Marolf, Counter-term charges generate bulk symmetries, Phys. Rev. D 72 (2005) 104025 [hep-th/0503105] [INSPIRE].

[12] I. Papadimitriou and K. Skenderis, Thermodynamics of asymptotically locally AdS spacetimes, JHEP 08 (2005) 004 [hep-th/0505190] [INSPIRE].

[13] D. Harlow and J.-Q. Wu, Covariant phase space with boundaries, JHEP 10 (2020) 146 [arXiv: 1906.08616] [INSPIRE].

[14] S. Carlip, Black hole entropy from conformal field theory in any dimension, Phys. Rev. Lett. 82 (1999) 2828 [hep-th/9812013] [INSPIRE].

[15] S.W. Hawking, M.J. Perry and A. Strominger, Soft hair on black holes, Phys. Rev. Lett. 116 (2016) 231301 [arXiv: 1601.00921] [InSPIRE].

[16] S. Haco, S.W. Hawking, M.J. Perry and A. Strominger, Black hole entropy and soft hair, JHEP 12 (2018) 098 [arXiv:1810.01847] [INSPIRE].

[17] L.-Q. Chen, W.Z. Chua, S. Liu, A.J. Speranza and B.d.S.L. Torres, Virasoro hair and entropy for axisymmetric Killing horizons, Phys. Rev. Lett. 125 (2020) 241302 [arXiv: 2006.02430] [INSPIRE].

[18] V. Chandrasekaran and A.J. Speranza, Anomalies in gravitational charge algebras of null boundaries and black hole entropy, JHEP 01 (2021) 137 [arXiv:2009.10739] [INSPIRE].

[19] E.E. Flanagan, Order-unity correction to Hawking radiation, Phys. Rev. Lett. 127 (2021) 041301 [arXiv: 2102 .04930] [INSPIRE].

[20] E.E. Flanagan, Infrared effects in the late stages of black hole evaporation, JHEP 07 (2021) 137 [arXiv:2102.13629] [INSPIRE].

[21] S. Pasterski and H. Verlinde, HPS meets AMPS: how soft hair dissolves the firewall, JHEP 09 (2021) 099 [arXiv: 2012.03850] [inSPIRE].

[22] W. Donnelly and L. Freidel, Local subsystems in gauge theory and gravity, JHEP 09 (2016) 102 [arXiv: 1601.04744 ] [INSPIRE].

[23] M. Geiller, Edge modes and corner ambiguities in 3d Chern-Simons theory and gravity, Nucl. Phys. B 924 (2017) 312 [arXiv: 1703.04748] [InSPIRE].

[24] A.J. Speranza, Local phase space and edge modes for diffeomorphism-invariant theories, JHEP 02 (2018) 021 [arXiv:1706. 05061] [INSPIRE].

[25] M. Geiller, Lorentz-diffeomorphism edge modes in 3d gravity, JHEP 02 (2018) 029 [arXiv: 1712.05269] [INSPIRE].

[26] M. Geiller and P. Jai-akson, Extended actions, dynamics of edge modes, and entanglement entropy, JHEP 09 (2020) 134 [arXiv:1912.06025] [INSPIRE].

[27] L. Freidel, M. Geiller and D. Pranzetti, Edge modes of gravity. Part I. Corner potentials and charges, JHEP 11 (2020) 026 [arXiv:2006.12527] [INSPIRE]. 
[28] W. Donnelly, L. Freidel, S.F. Moosavian and A.J. Speranza, Gravitational edge modes, coadjoint orbits, and hydrodynamics, JHEP 09 (2021) 008 [arXiv:2012.10367] [INSPIRE].

[29] J.D. Brown and J.W. York, Jr., Quasilocal energy and conserved charges derived from the gravitational action, Phys. Rev. D 47 (1993) 1407 [gr-qc/9209012] [INSPIRE].

[30] R.M. Wald and A. Zoupas, A general definition of 'conserved quantities' in general relativity and other theories of gravity, Phys. Rev. D 61 (2000) 084027 [gr-qc/9911095] [INSPIRE].

[31] V. Chandrasekaran, É.É. Flanagan, I. Shehzad and A.J. Speranza, A general framework for gravitational charges and holographic renormalization, arXiv:2111.11974 [INSPIRE].

[32] V. Balasubramanian and P. Kraus, A stress tensor for anti-de Sitter gravity, Commun. Math. Phys. 208 (1999) 413 [hep-th/9902121] [INSPIRE].

[33] R.C. Myers, Stress tensors and Casimir energies in the AdS/CFT correspondence, Phys. Rev. D 60 (1999) 046002 [hep-th/9903203] [INSPIRE].

[34] S. de Haro, S.N. Solodukhin and K. Skenderis, Holographic reconstruction of space-time and renormalization in the AdS/CFT correspondence, Commun. Math. Phys. 217 (2001) 595 [hep-th/0002230] [INSPIRE].

[35] M. Henneaux, Zero Hamiltonian signature spacetimes, Bull. Soc. Math. Belg. Ser. A 31 (1979) 47.

[36] C. Duval, G.W. Gibbons and P.A. Horvathy, Conformal Carroll groups, J. Phys. A 47 (2014) 335204 [arXiv: 1403.4213] [INSPIRE].

[37] C. Duval, G.W. Gibbons, P.A. Horvathy and P.M. Zhang, Carroll versus Newton and Galilei: two dual non-Einsteinian concepts of time, Class. Quant. Grav. 31 (2014) 085016 [arXiv: 1402.0657] [INSPIRE].

[38] C. Duval, G.W. Gibbons and P.A. Horvathy, Conformal Carroll groups and BMS symmetry, Class. Quant. Grav. 31 (2014) 092001 [arXiv:1402.5894] [INSPIRE].

[39] M. Henneaux and P. Salgado-ReboLledó, Carroll contractions of Lorentz-invariant theories, JHEP 11 (2021) 180 [arXiv:2109.06708] [INSPIRE].

[40] K. Parattu, S. Chakraborty, B.R. Majhi and T. Padmanabhan, A boundary term for the gravitational action with null boundaries, Gen. Rel. Grav. 48 (2016) 94 [arXiv:1501.01053] [INSPIRE].

[41] L. Lehner, R.C. Myers, E. Poisson and R.D. Sorkin, Gravitational action with null boundaries, Phys. Rev. D 94 (2016) 084046 [arXiv: 1609.00207] [INSPIRE].

[42] F. Hopfmüller and L. Freidel, Gravity degrees of freedom on a null surface, Phys. Rev. D 95 (2017) 104006 [arXiv:1611.03096] [INSPIRE].

[43] R. Oliveri and S. Speziale, Boundary effects in general relativity with tetrad variables, Gen. Rel. Grav. 52 (2020) 83 [arXiv: 1912.01016] [InSPIRE].

[44] S. Aghapour, G. Jafari and M. Golshani, On variational principle and canonical structure of gravitational theory in double-foliation formalism, Class. Quant. Grav. 36 (2019) 015012 [arXiv: 1808.07352] [INSPIRE].

[45] G. Jafari, Stress tensor on null boundaries, Phys. Rev. D 99 (2019) 104035 [arXiv: 1901.04054] [INSPIRE].

[46] L. Donnay and C. Marteau, Carrollian physics at the black hole horizon, Class. Quant. Grav. 36 (2019) 165002 [arXiv: 1903.09654] [INSPIRE]. 
[47] M. Mars and J.M.M. Senovilla, Geometry of general hypersurfaces in space-time: junction conditions, Class. Quant. Grav. 10 (1993) 1865 [gr-qc/0201054] [InSPIRE].

[48] R. Geroch, Asymptotic structure of space-time, in Asymptotic structure of space-time, F.P. Esposito and L. Witten eds., Plenum Press, New York, NY, U.S.A. (1977).

[49] A. Ashtekar, Asymptotic quantization: based on 1984 Naples lectures, Bibliopolis, Naples, Italy (1987).

[50] V. Chandrasekaran, É.É. Flanagan and K. Prabhu, Symmetries and charges of general relativity at null boundaries, JHEP 11 (2018) 125 [arXiv:1807.11499] [INSPIRE].

[51] G. Daŭtcourt, Characteristic hypersurfaces in general relativity. I, J. Math. Phys. 8 (1967) 1492.

[52] E. Gourgoulhon and J.L. Jaramillo, A 3+1 perspective on null hypersurfaces and isolated horizons, Phys. Rept. 423 (2006) 159 [gr-qc/0503113] [inSPIRE].

[53] T. Damour, Surface effects in black-hole physics, in Marcel Grossmann meeting: general relativity, (1982), pg. 587.

[54] G.A. Burnett and R.M. Wald, A conserved current for perturbations of Einstein-Maxwell space-times, Proc. Roy. Soc. Lond. A 430 (1990) 57.

[55] A.M. Grant, K. Prabhu and I. Shehzad, The Wald-Zoupas prescription for asymptotic charges at null infinity in general relativity, arXiv:2105.05919 [INSPIRE].

[56] R.M. Wald, On identically closed forms locally constructed from a field, J. Math. Phys. 31 (1990) 2378.

[57] E. Witten, Anti-de Sitter space and holography, Adv. Theor. Math. Phys. 2 (1998) 253 [hep-th/9802150] [INSPIRE].

[58] R.B. Mann and D. Marolf, Holographic renormalization of asymptotically flat spacetimes, Class. Quant. Grav. 23 (2006) 2927 [hep-th/0511096] [INSPIRE].

[59] Y. Kosmann-Schwarzbach and B.E. Schwarzbach, The Noether theorems: invariance and conservation laws in the twentieth century, Springer, New York, NY, U.S.A. (2011).

[60] T. Jacobson, Initial value constraints with tensor matter, Class. Quant. Grav. 28 (2011) 245011 [arXiv: 1108.1496] [INSPIRE].

[61] M.D. Seifert and R.M. Wald, A general variational principle for spherically symmetric perturbations in diffeomorphism covariant theories, Phys. Rev. D 75 (2007) 084029 [gr-qc/0612121] [INSPIRE].

[62] M. Henningson and K. Skenderis, The holographic Weyl anomaly, JHEP 07 (1998) 023 [hep-th/9806087] [INSPIRE].

[63] S. Hollands, A. Ishibashi and D. Marolf, Comparison between various notions of conserved charges in asymptotically AdS-spacetimes, Class. Quant. Grav. 22 (2005) 2881 [hep-th/0503045] [INSPIRE].

[64] J. de Boer, J. Hartong, N.A. Obers, W. Sybesma and S. Vandoren, Perfect fluids, SciPost Phys. 5 (2018) 003 [arXiv: 1710.04708] [InSPIRE].

[65] X. Bekaert and K. Morand, Connections and dynamical trajectories in generalised Newton-Cartan gravity II. An ambient perspective, J. Math. Phys. 59 (2018) 072503 [arXiv: 1505. 03739] [INSPIRE].

[66] L. Ciambelli, R.G. Leigh, C. Marteau and P.M. Petropoulos, Carroll structures, null geometry and conformal isometries, Phys. Rev. D 100 (2019) 046010 [arXiv:1905.02221] [INSPIRE]. 
[67] A. Bagchi, A. Mehra and P. Nandi, Field theories with conformal Carrollian symmetry, JHEP 05 (2019) 108 [arXiv:1901.10147] [INSPIRE].

[68] J. Hartong, Holographic reconstruction of 3D flat space-time, JHEP 10 (2016) 104 [arXiv: 1511.01387] [INSPIRE].

[69] L. Ciambelli and C. Marteau, Carrollian conservation laws and Ricci-flat gravity, Class. Quant. Grav. 36 (2019) 085004 [arXiv: 1810.11037] [INSPIRE].

[70] L. Ciambelli, C. Marteau, A.C. Petkou, P.M. Petropoulos and K. Siampos, Covariant Galilean versus Carrollian hydrodynamics from relativistic fluids, Class. Quant. Grav. 35 (2018) 165001 [arXiv:1802.05286] [INSPIRE].

[71] L. Ciambelli, C. Marteau, A.C. Petkou, P.M. Petropoulos and K. Siampos, Flat holography and Carrollian fluids, JHEP 07 (2018) 165 [arXiv:1802.06809] [INSPIRE].

[72] J.D. Brown, S.R. Lau and J.W. York, Jr., Energy of isolated systems at retarded times as the null limit of quasilocal energy, Phys. Rev. D 55 (1997) 1977 [gr-qc/9609057] [INSPIRE].

[73] I.S. Booth, Metric based Hamiltonians, null boundaries, and isolated horizons, Class. Quant. Grav. 18 (2001) 4239 [gr-qc/0105009] [INSPIRE].

[74] F. Hopfmüller and L. Freidel, Null conservation laws for gravity, Phys. Rev. D 97 (2018) 124029 [arXiv: 1802.06135] [INSPIRE].

[75] R.M. Wald, Black hole entropy is the Noether charge, Phys. Rev. D 48 (1993) R3427 [gr-qc/9307038] [INSPIRE].

[76] S. Bhattacharyya, V.E. Hubeny, S. Minwalla and M. Rangamani, Nonlinear fluid dynamics from gravity, JHEP 02 (2008) 045 [arXiv:0712.2456] [INSPIRE].

[77] M. Rangamani, Gravity and hydrodynamics: lectures on the fluid-gravity correspondence, Class. Quant. Grav. 26 (2009) 224003 [arXiv:0905.4352] [INSPIRE].

[78] R.F. Penna, BMS 3 invariant fluid dynamics at null infinity, Class. Quant. Grav. 35 (2018) 044002 [arXiv: 1708.08470] [INSPIRE].

[79] L. Ciambelli, C. Marteau, P.M. Petropoulos and R. Ruzziconi, Gauges in three-dimensional gravity and holographic fluids, JHEP 11 (2020) 092 [arXiv: 2006.10082] [INSPIRE].

[80] R. Fareghbal and A. Naseh, Flat-space energy-momentum tensor from BMS/GCA correspondence, JHEP 03 (2014) 005 [arXiv: 1312.2109] [INSPIRE].

[81] D. Kapec, P. Mitra, A.-M. Raclariu and A. Strominger, $2 D$ stress tensor for $4 D$ gravity, Phys. Rev. Lett. 119 (2017) 121601 [arXiv:1609.00282] [INSPIRE].

[82] É.É. Flanagan and D.A. Nichols, Conserved charges of the extended Bondi-Metzner-Sachs algebra, Phys. Rev. D 95 (2017) 044002 [arXiv:1510.03386] [INSPIRE].

[83] G. Compère, R. Oliveri and A. Seraj, The Poincaré and BMS flux-balance laws with application to binary systems, JHEP 10 (2020) 116 [arXiv: 1912.03164] [INSPIRE].

[84] S. Chakraborty and K. Parattu, Null boundary terms for Lanczos-Lovelock gravity, Gen. Rel. Grav. 51 (2019) 23 [Erratum ibid. 51 (2019) 47] [arXiv:1806.08823] [InSPIRE].

[85] W. Vogel, Über lineare Zusammenhänge in singulären Riemannschen Räumen (in German), Arch. Math. 16 (1965) 106.

[86] L. Ciambelli and R.G. Leigh, unpublished notes. 